\title{
A rotation in $0 \& G$ as a GP specialist trainee: What can you expect?
}

After a morning of introduction to a number of faces - sisters, matrons, the smiling consultants, one in a beige suit who looked like he did a lot of private practice and the other in scrubs who repeated our eight names again and again, it was time for work. After being shown how to use a speculum, we marched on with trepidation.

Two FY2s, Four GPSTs including myself, were starting the rotation. There were only two career STs already in their posts, in total seven girls and the token boy. The afternoon was spent in our respective rostered area - which we all duly left early and gathered on the gynae ward to do a ward round as none of the patients had been seen; a sure sign that newbies were about! Having realised that I had no previous O\&G experience - this was beginning to look easier. Most were postop patients not having problems with their heart or lungs, the routine questions were asked. 'Any pain? How's the bleeding? Passed urine? All fine, discharged!!'

'Now pull' - shocking how the SpR nearly squatted while yanking apart the rectus sheath! 'Show me the angles, follow me, keep perpendicular (while suturing). Congratulations, no return, no refunds!!' This I heard throughout the night at the Caesarean sections. The nice thing is, one walks around with a permanent smile after assisting with this amazing life event.

My real baptism of fire was with the weekend of nights. I had asked about specifics of history taking and management from friends who were in O\&G. Various cases were seen: pregnant with abdominal pain and PV bleeding, lost coil with possible perforation, menorrhagia for weeks with $\mathrm{a} \mathrm{Hb}$ of 5 and hyperemesis gravidarum. The examination was a painful ordeal; forgot the light, forgot to take swabs, forgot to ballot uterus and so the night went on. When I did manage to feel a gravid uterus, my consultant's words 'like an apple' came to mind.

During the week I spent a morning in theatre assisting my consultant. There was a vaginal hysterectomy, anterior repair with sacrospinous fixation, and laparoscopy. The latter was interesting as the anatomy could be seen, but mainly involved moving a plastic tube in the cervix, anteriorly and posteriorly to antevert and retrovert the uterus. At the next theatre session I opted for gynaecology clinics. 'That is the way training is going,' a consultant said, 'We used to see SHOs in theatre all the time, now we hardly see any!!' Perhaps this is due to the fact of reduced numbers of career O\&G SHOs and more GP trainees taking up the places.

In clinic, I saw conditions that GPs face and are required to manage, such as management of bleeding on the pill, what is considered to be a normal menstrual cycle, what irregular cycles mean, referrals for intermenstrual/post-menopausal bleeding and management of incontinence. SHOs are supernumery and benefit most by seeing patients and discussion with consultants.

Labour ward on call was spent cowering behind the registrar. 'Perhaps your SHO could deal with this delivery' was received by myself with a blank look and 'I do not know how to deliver a baby - let alone if a shoulder became stuck!' On the whole I think I speak for the majority of new entrants to O\&G when I say that obstetrics is poorly studied at medical school, being seen as a specialised SpR led field. The physiology of labour, stages of labour, abnormal CTGs and when Caesarean sections are indicated seems a mystical area. On calls also meant seeing patients on the postnatal ward, carrying out postop checks, and completing discharge letters. As $\mathrm{SHOs}$ we dealt with antibiotics for wound infections, titration of blood pressure medication, iron prescriptions for low $\mathrm{Hb}$, drains and urine output.

Antenatal clinics comprised discussion of vaginal birth after Caesarean section (VBAC), twin pregnancies, and seeing patients with comorbidities such as diabetes and thyroid disorders. Complications of pregnancy such as preeclampsia and cholestasis were also focused on.

Overall, I think this is a dynamic field with an interesting mix of surgery and medicine. It requires compassion and can be stressful dealing with unhappy events such as miscarriage. Hopefully this article has given you an idea of what this rotation is like - especially from a GPST perspective and encourage you to take this rotation up in your foundation/ST years.

\section{Sivashankari Thiagamoorthy}

DOI: 10.3399/bjgp09X407018 